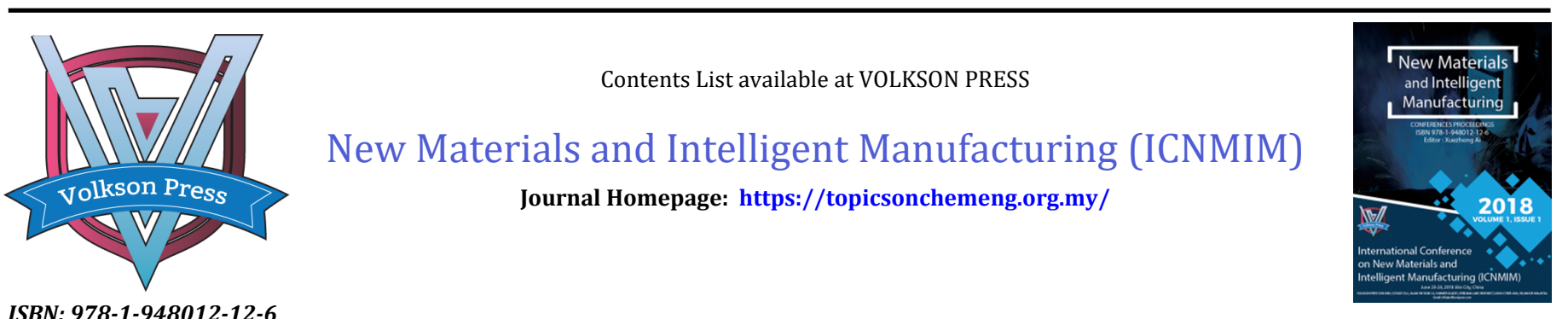

ISBN: 978-1-948012-12-6

\title{
PROPERTIES OF DIFFERENT COMPONENT STARCH PLASTICIZERS AND REINFORCEMENT OF PLASTICIZED STARCH FIBERS
}

\author{
Hou Zhesheng ${ }^{1}$, Qiu Bofeng ${ }^{1}$ and Yin Jinghua ${ }^{2 *}$ \\ ${ }^{1}$ College of Mechanical \&ElectricalEngineering, Jilin Institute of Chemical Technology, Chengde Street, Jilin City, China \\ ${ }^{2}$ Changchun Institute of Applied Chemistry, Chinese Academy of Science,RenminStreet,Changchun City, China \\ *Corresponding Author E-mail: 13844675398@126.com
}

This is an open access article distributed under the Creative Commons Attribution License, which permits unrestricted use, distribution, and reproduction in any medium, provided the original work is properly cited

\section{ARTICLE DETAILS}

\section{Article History:}

Received 26 June 2018

Accepted 2 July 2018

Available online 1 August 2018

\section{ABSTRACT}

In this study, pullulanase was debranched in different amylose content starch and normal corn starch, and amylose with different chain length was separated by centrifugation. At the same time, the mechanical properties and application properties of the materials were studied. The higher the amylose content, the higher the tensile strength of the plasticized starch, the lower the water absorption after plasticization, and the better the water resistance; this study limited the plasticizer to be biocompatible. Degradation of glycerol in the body. The high linear content of plasticized starch is a new material with broad application prospects. Thermoplastic starch has hygroscopicity, but its mechanical properties are lower than other thermoplastic polymers. In this study, wood starch fiber modified starch was added, and it was concluded that natural wood fiber is a good reinforcing fiber for plasticized starch.

KEYWORDS

pullulanase debranching; High amylase starch; New material.

\section{INTRODUCTION}

As a widely studied and researched starch physical modification technology, starch plasticization is a technology that has been successfully implemented by the well-known foreign companies in the industrialization of products $[1,2]$

According to a study, the uniqueness of this research lies in the selection of natural starch with different amylose content, and the debranching of pullulanase from ordinary corn starch, and centrifugation of amylose with different chain lengths [3].

This chapter focuses on material samples prepared using different processing methods after plasticizing these different components of starch. At the same time, the mechanical properties and application properties of the materials were studied. Water is a very good plasticizer for starch. Because of its volatility, its plasticized starch cannot be used in an open environment. Non-volatile plasticizers are preferred for glycerol. This chapter studies the limitation of plasticizers to glycerol. Based on a study, another reason is that it is biocompatible, edible and in vivo [4].
Thermoplastic starch has hygroscopicity and its mechanical properties are lower than those of other thermoplastic polymers. Adding reinforcing materials may improve performance. It is a more appropriate strategy to add degradable polymers and natural polymers due to the polarity and degradability of starch. This chapter adds wood. Study showed pulp fiber modified starch [5].

Thermoplastic starch has hygroscopicity and its mechanical properties are lower than those of other thermoplastic polymers. Adding reinforcing materials may improve performance. It is a more appropriate strategy to add degradable polymers and natural polymers due to the polarity and degradability of starch. This chapter adds wood. Pulp fiber modified starch.

\section{MATERIALS AND METHOD}

The raw materials used in this study were different starches, debranched amylose, and plasticizers.

\begin{tabular}{|lll|}
\hline Sample & Content & Source \\
\hline Waxy corn starch & amylose $4 \%$ & Changchun Dacheng Industrial Group \\
Normal corn starch & amylose $27 \%$ & Changchun Dacheng Industrial Group \\
High amylose corn starch & amylose $55 \%$ & U.S. National Starch \\
Pullulan starch & $/$ & Lab made \\
Wood pulp fiber & Cellulose $98 \%$ & Jixi Chemical Fiber \\
Glycerol & Analytical purity & Xiqiao Chemicals \\
\hline
\end{tabular}

2.1 Preparation of Different Component Starch Plasticized Material Spline

\subsubsection{Preparation of Plasticized Starch Film Pressed Sheets}

After premixing in a high-speed mixer, they were blended in a HAAKE PolyLab co-rotating twin-screw extruder. The temperature of each section of the twin-screw extruder was $80 \mathrm{rpm}$ and the residence time of the material in the twin-screw extruder was approximately 120 seconds After twin-screw blending, granulation was performed. The blended sample was placed in a polyimide film sandwich and hot-pressed at $150^{\circ}$ C. and $10 \mathrm{MPa}$ for 5 minutes on a plate vulcanizing machine, and then the 
spline was promptly placed on another plate. On the vulcanizing machine, natural cooling to room temperature at $10 \mathrm{Mpa}$, according to national standards made spline, each prepared into $3,50.0 \mathrm{~mm} \times 8.6 \mathrm{~mm} \times 1.0 \mathrm{~mm}$ (neck width 4.0mm) spline [6].

\begin{tabular}{|cll|}
\hline Sample No. & Starch content & Plasticizer content \\
\hline $1 \#$ & Waxy corn starch 100 pcs & glycerin 30 pcs \\
$2 \#$ & Normal corn starch 100 pcs & glycerin 30 pcs \\
$3 \#$ & High amylose corn starch 100 pcs & glycerin 30 pcs \\
\hline
\end{tabular}

\subsubsection{Preparation of component separation starch film}

watch glass, dried at 45 degrees for 24 hours, and vacuum dried for 4

Starch: Water: Glycerine = 1:5: $(0 \sim 0.3)$, cast on a $10 \mathrm{~cm}$ diameter PTFE

\begin{tabular}{|clcc|}
\hline Sample No. & \multicolumn{1}{c|}{ Starch content } & Moisture content & Glycerol content \\
\hline $4 \#$ & $\begin{array}{l}\text { One part of ordinary starch centrifugal } \\
\text { upper layer }\end{array}$ & 5 Pcs & 0.3 Pcs \\
& $\begin{array}{l}4 \# \quad \text { centrifuging the lower layer, } \\
\text { enzymatically centrifuging the upper } \\
\text { layer 1 part }\end{array}$ & 5 Pcs & 0.3 Pcs \\
\hline
\end{tabular}

\subsubsection{Preparation of Plasticized Starch and Plant Fiber Reinforced Plastic Injection Spline}

The natural wood fiber pulp boards were pulverized and opened by a pulverizer, glycerin infiltrated for 12 hours, starch: glycerin: wood pulp fiber $=100: 40: 3$ high-speed mixing, twin-screw blending granulation, injection molding machine for injection stretching and impact spline. The comparative spline is starch: glycerol $=100: 30$, and additionally molded into a film such as 4\# [7].

\begin{tabular}{|cllc|}
\hline $\begin{array}{c}\text { Sample } \\
\text { No. }\end{array}$ & \multicolumn{1}{c}{$\begin{array}{l}\text { Starch } \\
\text { content }\end{array}$} & $\begin{array}{l}\text { Moisture } \\
\text { content }\end{array}$ & $\begin{array}{l}\text { Wood fiber } \\
\text { content }\end{array}$ \\
\hline $6 \#$ & $\begin{array}{l}\text { Normal corn } \\
\text { starch 100 Pcs }\end{array}$ & 30 Pcs & 0 \\
$7 \#$ & $\begin{array}{l}\text { Normal corn } \\
\text { starch 100 Pcs }\end{array}$ & 40 Pcs & 3 \\
\hline
\end{tabular}

\subsection{Different component starch material mechanics test}

The mechanical properties were measured with a universal material testing machine. The INSTRON 1121 universal material testing machine was produced by the American INSTRON company. The specific parameters were: the maximum test force was $100 \mathrm{kN}$, the indication accuracy was $\pm 0.5 \%$, the sampling rate was $10 \mathrm{~Hz}$, and the tensile force was at a speed of $10 \mathrm{~mm} / \mathrm{min}$, three splines were tested for each sample [8].

\subsection{Water resistance and oil resistance test}

The water resistance performance was measured by immersing in deionized water at room temperature and changing the weight and using the formula $=($ membrane quality after moisture absorption - dry film quality) / dry film quality* $100 \%$ to calculate the water uptake.

Cut a certain amount of film and weigh it to W0. Soak in salad oil at room temperature. Remove the film from the surface with filter paper at regular intervals. Weigh it to W1, then the oil absorption rate of the film. The formula is: oil absorption $=($ film quality after oil absorption - dry film quality) / dry film quality* $100 \%$ [9].

\subsection{Water contact angle test}

The spin measurement is based on the fixed drop theory and is determined by the measurement and analysis system of a contact angle meter (DSA 100 KRUSS GMBH, Hamburg). The measurement conditions are: room temperature, six measurements per sample. The static contact angle of the sample was measured by the sessile drop method. $2 \mu \mathrm{L}$ droplets of water were dropped onto the surface of the film. The droplet surface was quickly captured with a video camera. The computer was then fitted to calculate the static contact angle of the sample. After removing the maximum and minimum values, calculate the average value and standard deviation.

\subsection{Starch FTIR Test}

The surface of the sample was characterized by ATR-FTIR using a Bruker Vertex 70 Fourier Transform Infrared Spectrometer (FTIR). The reflective element of the instrument was $\mathrm{ZnSe}$. The bottom angle of the test was $45^{\circ}$, the resolution was $2 \mathrm{~cm}-1$, and the internal reflection angle was $45^{\circ}$. Analysis of the Mechanism of Action of Different Components of Plasticized Plasticized Starch.

\section{ANALYSIS OF MECHANICAL PROPERTIES OF MATERIALS}

Figures 1(a) to (f) show the plasticized starches of different compositions. Figure $1(\mathrm{~g})$ shows the mechanical properties of modified starch films. From this figure, it can be seen that as the amylose content increases, the tensile strength increases, which may be due to the action of amylose, and the higher purity amylose has better film forming properties.

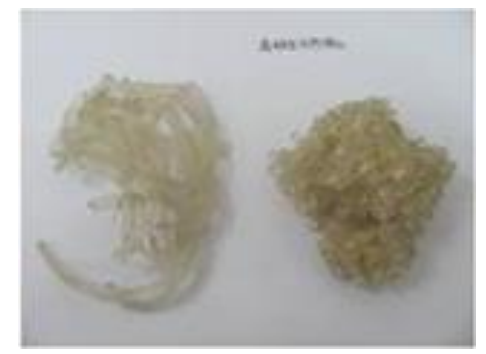

Figure 1(a): Plasticizing waxy starch granules 


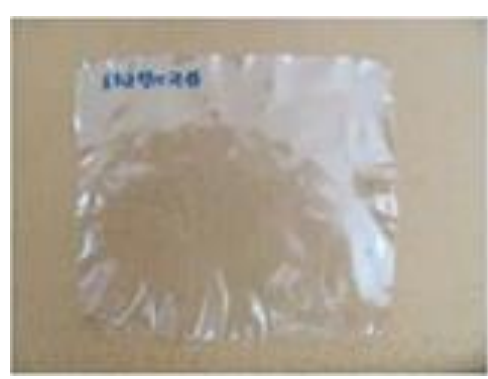

Figure 1(b): Plasticizing waxy starch film

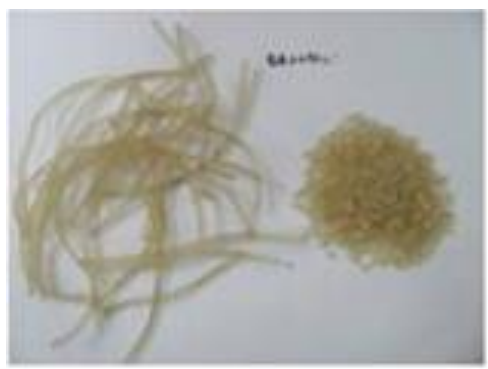

Figure 1(c): Plasticizing waxy starch granules

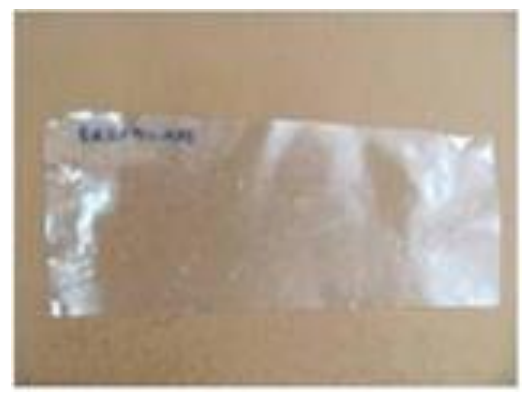

Figure 1(d): Plasticizing corn starch film

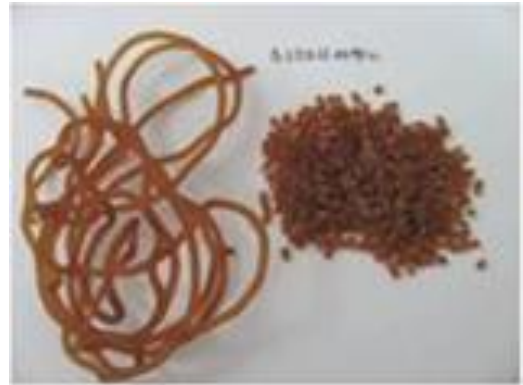

Figure 1(e): Plasticizing amylose granules

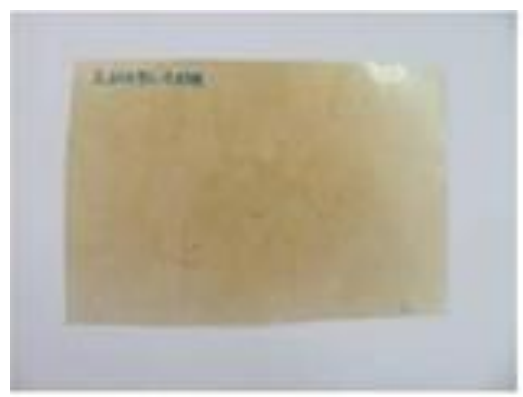

Figure 1(f): Plasticizing high amylose film

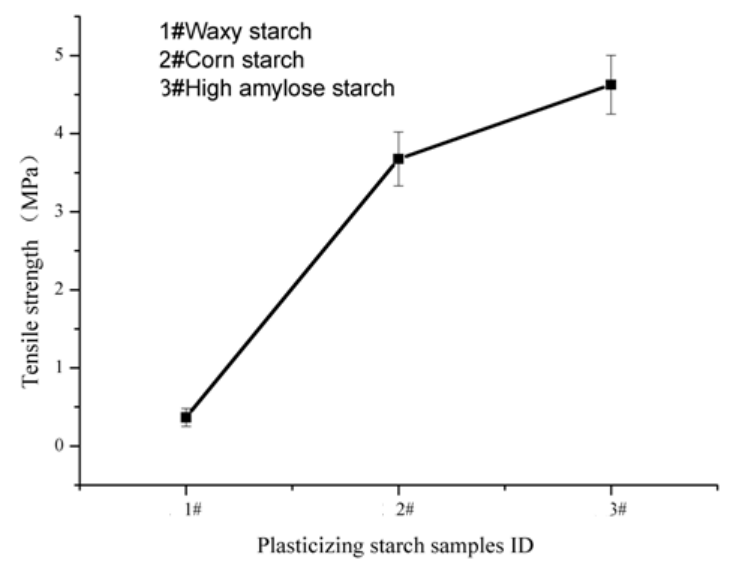

Figure 1(g): Variation of the plastic starch tensile strength with the content of amylose

Figures 2(a) and (b) show the 3\% natural wood cellulose fiber reinforced plasticized normal starch injection molded bar. Figure shows the change of the tensile strength of the spline, the tensile strength increases from $1.38 \mathrm{MPa}$ to $1.99 \mathrm{MPa}$, and the tensile strength increases $44.2 \%$. This is mainly due to the fact that natural fibers and starch have the same monomer structure, plasticizers have a plasticizing effect on both, and plasticized starch and plasticized fibers have better compatibility.

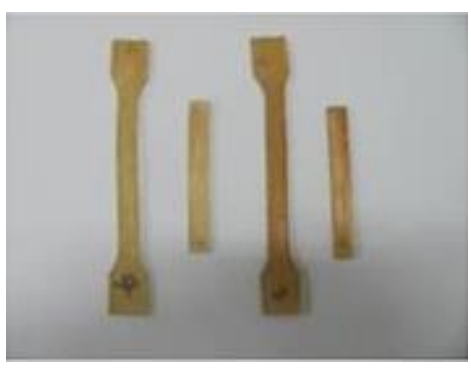

Figure 2(a): Plasticizing starch injection sample

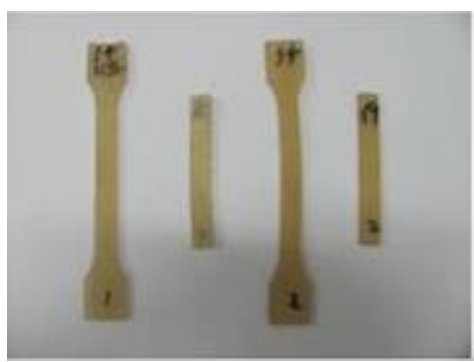

Figure 2(b): Plasticizing starch added wood fiber injection sample

\subsection{Analysis of water resistance and oil resistance of plasticized} starch film

Figure 3(a) shows the water absorption of the starch film. It can be seen from the figure that with the increase of the amylose content, the water absorption of the plasticized starch film is significantly reduced, and the amylose starch has better water absorption resistance [10].

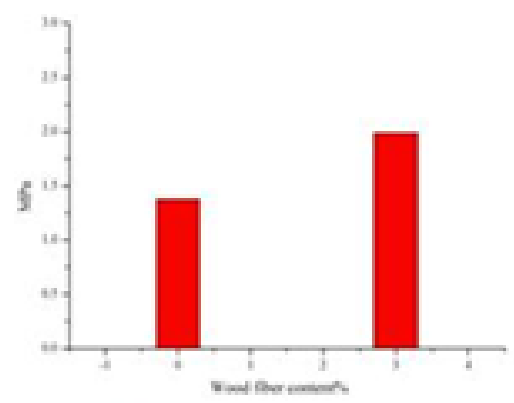

Figure 3(a): Variation of the tensile strength with the content of wood fiber 


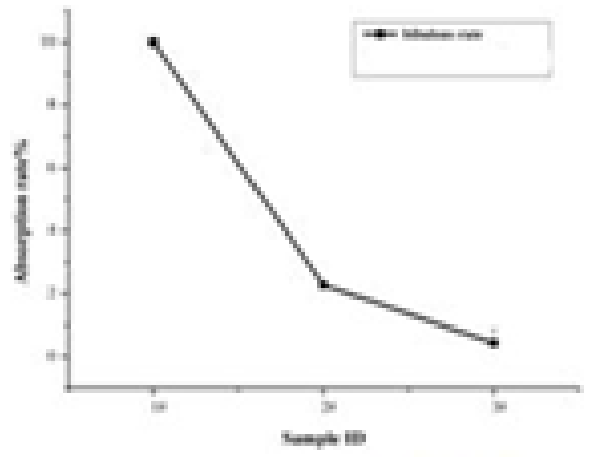

Figure 3(b): Variation of absorption rate with amylose contents

\subsection{Hydrophilicity analysis}

It can be seen from Figure 3(b) that the contact angle increases with the increase of the amylose content, and the contact angle is affected by both the processing method and the surface polarity of the molded material. Increasing the polarity of the molded material may increase the surface polarity of the plasticized starch. It can be predicted that with the increase of amylose content, the contact angle may change from hydrophilic to hydrophobic over $90^{\circ}$, which indicates that the polarity changes and there is a polarity to non-polarity transition. If blended with non-polar materials, the compatibility will increase.

\subsection{Analysis of different components of starch glycerol plastication mechanism}

Figure 4(b) Infrared spectrum of plasticized starch of different components, with a hydroxyl peak at 3300. In the case of internal standard, red common starch and green fiber increase the normal starch infrared coincidence, and black amylopectin also basically coincides with the upper curve; high amylose Decreased, short straight-chain and long amylose significantly reduced, among which pink long amylose was the lowest, indicating the amount of hydroxyl-containing, the lowest is not easily plasticized, and it is not easy to absorb water. The main reason may be that there is a helical structure in amylose, and the structure of hydrogen bonds in the helix is stable and the infrared is not obvious.

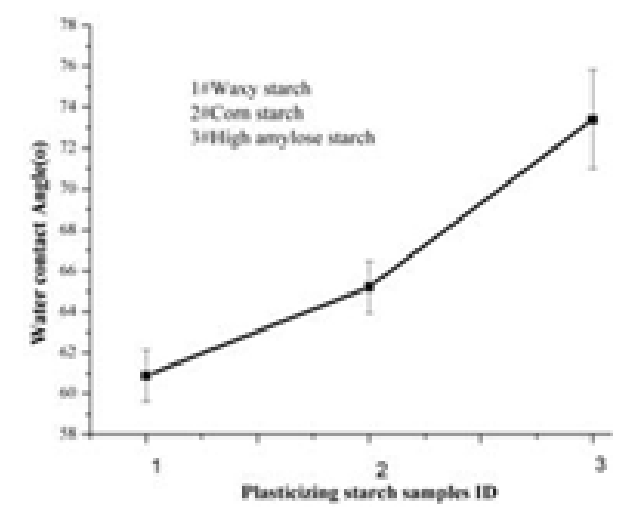

Figure 4 (a): Different components of the plastic starch water contact Angle

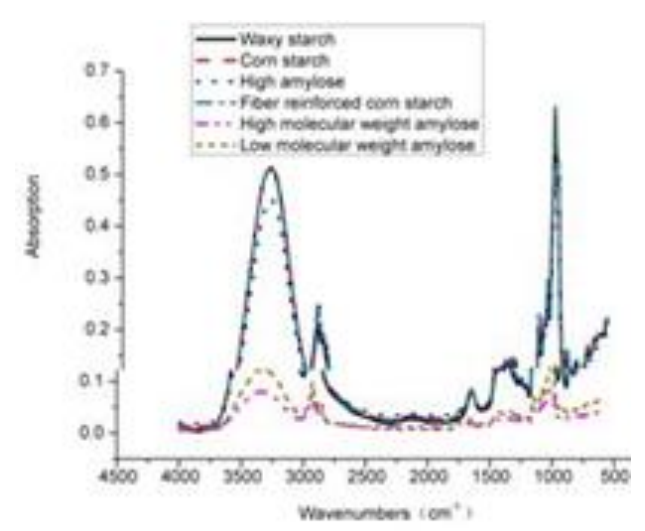

Figure 4 (b): Different components of the infrared spectrum plastic starch figure

\section{CONCLUSION}

The tensile strength of glycerin plasticized starch with different components was different. The higher the amylose content, the higher the tensile strength of the plasticized starch. The water absorption of the different components of glycerol plasticized starch is quite different. The higher the amylose content, the lower the water absorption after plasticization and the better water resistance; the plasticized starch has good oil resistance, and the straight chain High starch content, relatively high oil resistance. Natural wood fiber is a good reinforcing fiber for plasticized starch, $3 \%$ added to increase the tensile strength of injection molded spline $40 \%$.

\section{ACKNOWLEDGMENTS}

Fund Project: Significant Scientific and Technical Project in Jilin (20170201003GX)

\section{REFERENCES}

[1] Nanda, P.K., Rao, K.K., Nayak, P.L. 2007. Biodegradable polymers. XI. Spectral, thermal, morphological, and biodegradability properties of environment-friendly green plastics of soy protein modified with thiosemicarbazide. Journal of Applied Polymer Science, 103 (5), 31343142 .

[2] Tetlow, I.J. 2011. Starch biosynthesis in developing seeds. Seed Science Research, 21 (1), 5-32.

[3] Pushpadass, H.A., Marx, D.B., Hanna, M.A. 2008. Effects of Extrusion Temperature and Plasticizers on the Physical and Functional Properties of Starch Films. Starch-Starke, 60 (10), 527-538.

[4] Mccready, R.M., Hassid, W.Z. 1943. The Separation and Quantitative Estimation of Amylose and Amylopectin in Potato Starch. Journal of the American Chemical Society, 65 (6), 1154-1157.

[5] Kitamura, S.K. 2010. Biodegradable articles obtained from enzymatically synthesized amylose.

[6] Liu, P., Yu, L., Wang, X.Y. 2010. Glass transition temperature of starches with different amylose/amylopectin ratios. Journal of Cereal Science, 51 (3), 388-391.

[7] Shogren, R. 2007. Effect of Orientation on the Physical Properties of Potato Amylose and High-Amylose Corn Starch Films. Biomacromolecules, 8 (11), 3641-3645.

[8] Marques, A.P., Reis, R.L., Hunt, J.A. 2002. The biocompatibility of novel starch-based polymers and composites: in vitro studies. Biomaterials, 23 (6), 1471-1478.

[9] Kui, D., Qin, L., Yakun, B., Kai, M., Wang, W.J., Yuan, D.Q., Wang, Y.B. 2016. High surface area porous polymer frameworks: Potential host material for lithium-sulfur batteries. Journal of Alloys and Compounds, 657.

[10] Tan, K.J., Yu, Z.B. 2014. Preparation and Characterization of Scalable and Multi-Functional High Conductivity Polymer Electrode Material. Advanced Materials Research, 3043 (898). 